\title{
Some Reflections on the Mechanization of Mental Labor in the Computer Age ${ }^{1)}$
}

\author{
Wu Wen-tsun \\ Institute of System Sciences, AMSS, Academia Sinica \\ Beijing 100080, China \\ (xgao,mli)@mmrc.iss.ac.cn
}

The mankind is now entering a new era of information age or computer age characterized in particular by the presence of the powerful tool of computers. Before we consider how the mankind should deal with and act in this coming era, let us first give a brief reflection of what the mankind had thought about and had act in the past era of industrial revolutions accompanied by technological revolutions. Being a mathematician, the present author would like to present some of such reflections from a mathematical point of view. In fact, the novel creations of coordinate geometry by R.Descartes (1596-1650) and P.Fermat (1601-1665) as well as the infinitesimal calculus by I.Newton (1642-1665) and G.W.Leibniz (1646-1716) in 17th century made possible the study of movements and variations in a quantitave way. It provided thus powerful tools to nourish and impede the coming revolutions in industry and technology.

The past era of industrial revolutions may be roughly subdivided into 3 stages. The first stage, from 60th and 70th of 18th century onwards, was accompanied by the invention of Watt's vapor machine, the sewing machine, etc. in industry. Mathematicians, being intoxicated by the wonderful powerfulness and wide applicability of the newly invented method of calculus, were delighted to indulge themselves to such applications like improving of manufacturies, building of ships and even constructions of frigates, etc. Mathematics was itself benefitted in that calculus had grown up to become a giant discipline of mathematical analysis. Among the mathematicians involved in this movement we may cite particularly:

D'Alembert (1717-1783), G.Monge (1746-1810), L.Euler (1707-1783), J.L.Lagrange (17361813), P.S..Laplace (1749-1827), etc.

The second stage may be considered to be roughly from 60 th of 19 th century onwards. In technology it was characterized by the invention of electric machine, electric motor, electric telegraphy, etc. Among mathematicians-physicists we may cite e.g.: G.Green (1793-1841) and S.D.Poisson (1791-1840) for the creation of potential theory, C.F.Gauss (1777-1855) for the study of magnetism and invention of telegraphy, and J.C.Maxwell (1831-1879) for the establishment of electro-magnetism theory and equations.

The third stage was roughly from 40th of 20th century onwards. It was accompanied by exciting achievements in the revolution of technologies: The discoveries and inventions of computers, atomic energies, satellites, production automation, genetic engineering, superconductivity, lasers, etc. In mathematics and related fields there appeared a lot of entirely

The present paper was originally for the International Symposium on Frontiers in Science in celebration of 80 Birthday of Professor C.N.Yang taken place in Tsing-Hua University, 17-19 June, 2002. 
new disciplines which played important roles, direct or indirect, for the development of new industries and technologies: Mathematical logic, numerical analysis, topology, functional analysis, relativity theory, quantum mechanics, game theory, cybernetics, statistics, stochastic processes, mathematical biology, etc. Among the giant figures of mathematicians we may cite e.g. D.Hilbert (1862-1948), H.Poincaré (1854-1912), R.A.Fisher (18901062), A.H.Kolmogorov (1903-1087), J. von Neumann (1903-1957), A.Einstein (1879-1955), N.Wiener (1894-1964), and many others.

In order to get a concise general idea of what happens in the past era of industrial revolutions and to predict what will happen in the coming new era, let us cite some paragraphs from the classic $\ll$ Cyberenetics $(1948) \gg$ of N.Wiener as given below:

"Perhaps I may clarify the historical background of the present situation if I say that the first industrial revolution was the devaluation of the human arm by the competition of machinery.

The modern industrial revolution is similarly bound to devalue the human brain at least in its simpler and more routine decisions."

The present author would like to replace the above ideas of Wiener by some alike statements as given below:

The era of past industrial revolutions is characterized by the lessening or even replacing of physical labors by some kinds of machineries.

The coming era of new industrial revolutions will be characterized by the lessening or even replacing of mental labors by some new kinds of machineries.

Such new machineries do occur, e.g. the computers.

The appearance of computers in 40th of last century rendered the possibility of lessening or even replacing of mental labors by some machineries to be a reality. Thus, in the latter half of last century there appeared an active discipline of artificial intelligence. It deals with such intricate mental labors like language-translations, chess-playing, logical-reasoning, disease-diagnosis, etc. With various kinds of expert-systems the specialists in computers had been able to lessen the above-mentioned mental labors and many others, though yet far from being able to replace such mental labors by computers.

Mathematical activities are clearly some particularly instructive mental labor. So far mathematics was concerned, we may cite during its historical development some examples of lessening or reducing of mental labor, or in the terminology of the present author, mechanization of mental labor, as given below.

Ex.1. The replacement of highly difficult solving of arithmetic problems by solving of simple algebraic equations, as we learn in primary schools.

Ex.2. In the Song and Yuan Dynasties (9c-14c) of ancient China, the introduction of the notions Heaven Element, Earth Element, etc. rendered the previouly difficult problems of establishing equations from given datas almost a triviality.

Ex.3. In year 1614 J.Napier (1550-1617) introduced logarithms which replaced the laborious procedures of multiplication-division by the much easier procedures of additionsubtraction.

Ex.4. In 1637 the publication of the classic «La Géométrie 》 by Descartes rendered possible the replacement of the delicate geometry theorem-proving which requires genious- 
like talents by almost trivial, though complicate and laborious, algebraic manipulations.

Ex.5. In 1642 B.Pascal (1623-1662) invented computing machines for additions and in 1674 Leibniz improved to machines for additions and multiplications. These furnished concrete examples of replacing some kind of mental labors by some kind of machineries.

We would like to point out that, all leaping advances of mathematics in past times were actually accompanied by some kinds of mechanization of mathematics as mental labor, which may be seen clearly from above Exs.1 and 4.

We would like to point out that, the general idea of mechanization of mental labor, mathematics in particular, was well-possessed by such giant figures like Descartes and Leibniz. Below we shall give some of their sayings which are extracted from the well-known treatise $\ll$ Mathematical Thought from Ancient to Modern Times (1972) 》 by M.Kline:

"In his [Descartes'] view algebra mechanizes mathematics so thinking and processes become simple and do not require a great effort of the mind. Mathematical creation might become almost automatic."

"Even the logical principles and methods might be expressed symbolically and the whole system employed to mechanize all reasonings."

"The power of algebra to symbolize and even mechanize geometrical arguments had impressed both Descartes and Leibniz, ..., and Leibniz launched more ambitious program."

"[Leibniz] became impressed with the possibility of a broad calculus that would enable man to reason in all fields mechanically and effortlessly."

"The general science was to provide a rational, universal language that would be adapted to thinking. The concepts, ..., could be combined in an almost mechanical way."

Below is a list of important personages mainly mathematicians who had made important contributions to the further promotion of mechanization of mathematics as mental labor along the lines of thought of Descartes and Leibniz:

G.Boole (1815-1864): Establishment of Boolean algebra.

A.N.Whitehead (1861-1949) and B.Russell (1872-1970): Development of mathematical philosophy and publication of $\ll$ Principia Mathematica $(1910-1913) \gg$.

D.Hilbert (1862-1943): Founding of axiomatic method, establishment of mathematical logic and proof theory.

K.Goedel (1906-1978): Discovery of incompleteness theorem in 1931 which proves that True is not the same as Provable. This overthrew in particular the endeavor of Hilbert to prove the consistency of mathematics.

A.Turing (1912-1954): Description of a mathematical model named after him of computers.

J.Herbrand (1908-1931): Formulation of an ( incomplete ) algorithm of general theoremproving which was followed by most of mathematical logicians henceforth.

A.Tarski (1902-1983): First algorithm in 1950 for proving of theorems in elementary algebra and elementary geometry.

H.Wang (1921-1995): First succesful computer-proof in 1960 of all propositions in WhiteheadRussell's $\ll$ Principia Mathematica .

From the above we see that the general ideas as well as general methods of mechanization of mental labor, mainly mathematics as a representative one, were due to mathematicians including mathematical logicians. This is not an accidental matter. There are deep reasons 
to be so.

First of all let us remark that mathematics is a fundamental basis of, as well as has wide applicability to, actually all kinds of science and technology. It follows that mathematics, among all kinds of mental labors, should have the highest priority and greatest urgency to be mechanized.

What is more important is this: Mathematics, among all kinds of mental labors, is the easiest one to be mechanized. In fact, mathematics has the peculiarity of being precise, concise, exact, unambiguous, rigorous, etc., as compared with such mental labors treated by artificial intelligence. Owing to such superiority there are reasons to believe that mathematics will be much easier to be mechanized than other kinds of mental labors. It turns out that this is really so. The proving of theorems in euclidean geometry, as everybody learned in elementary schools, is such a kind of mental labor which requires intricate genious-like reasonings and thus seemingly far from being mechanizable. Remark however that geometrical theorems are all stated in precise, concise, exact, unambiguous, and rigorous way. In taking grant of the special characters of geometrical theorems involved, the present author had showed in late 70th of last century, how to prove the main part of geometrical theorems in an efficient algorithmic way. Hundreds of non-trivial theorems had been proved and even discovered in this way, as may be seen from the book of S.C.Chou, see [Ch] in the Bibliography at the end of this paper.

In view of what we have said above about the mechanization of mathematics in the era of industrial revolutions from 18th century onwards, we may put forth the following question:

Question Q: In the coming era of computer age, what the mathematians should do? In particular, what the Chinese mathematicians should do?

For answering the above question let us first remark that the appearance of computers had evolved a new discipline of computer science. According to the authority D.E.Knuth of computer science, computer science is actually a science of algorithms, cf. [K]. In fact, algorithms may be turned readily into programs. The computers, starting from the given datas, will run automatically in accordance to the instructions furnished by the program, and will give the answers required quite often in a finite time. This will give the way of mechanization of mental labor for the problems to be solved, as shown by the example of geometry theorem-proving mentioned above.

Now in contrast to the ancient Greek mathematics represented by Euclid's $\ll$ Elements of Geometry», the ancient Chinese mathematics is actually a kind of mathematics of algorithms. Most of the results in our ancient mathematics were expressed in the form of algorithms instead of theorems, contrary to the ancient Greek case. According to what D.E.Knuth had said, the ancient Chinese mathematics is actually a kind of computer science. The success of our geometry theorem-proving as described above is actually due to our detection of this point and our trials to follow the steps of our ancestors. The further development of mathematics-mechanization in following the line of thought of our ancestors may be seen from the writings of the present author and his colleagues as shown in the Bibliography at the end.

Our answer to above Question Q is now quite clear. 


\section{References}

[Ch] Chou,S.C., Mechanical Geometry Theorem-Proving, Reidel, 1988.

[K] Knuth, D.E., Computer Science and its Relation to Mathematics, Amer. Math. Monthly, 81 (1974) 323-343.

[MM] Mathematics-Mechanization Research Preprints, MM Reseach center, Inst. of Systems Science, CAS, 1-20 (1987-2001).

[Wu] Wu, W.T., Mathematics Mechanization, Mechanical Geometry Theorem-Proving, Mechanical Geometry Problem-Solving, and Polynomial Equations-Solving, Science Press / Kluwer Academic Publishers, 2000. 